\title{
Fault Insertion for Controller Calibration in a Range of Engine Models
}

\author{
Romain Gillot $^{*} \quad$ Alessandro Picarelli* ${ }^{*}$ Mike Dempsey* \\ ${ }^{*}$ Claytex Services Ltd, Edmund House, Rugby Road, Leamington Spa, CV32 6EL \\ \{romain.gillot, alessandro.picarelli, mike.dempsey\}@claytex.com
}

\begin{abstract}
This paper investigates the response of the Engine Control Unit (ECU) in relation to abnormal engine operation. Four different faults are introduced in a physical engine model at random times during simulation to observe the effects on the model results and system behaviour. It is then shown that the sensors used throughout the engine model are capable of detecting any type of fault using signals for quantities that would be measurable in a real engine. Finally, the soft ECU switches to emergency/limpo home operation and limits the engine performance to help prevent what could be mechanical damage in a real engine.
\end{abstract}

Keywords: $\quad$ engine, fault, ECU, detection

\section{Introduction}

Predictive modelling is becoming increasingly popular to dimension or calibrate systems prior to the prototype stage. If current models are capable of representing the behaviour of physical systems very accurately, they often only model the system's expected behaviour. Some particular tasks like Engine Control Unit (ECU) calibration require the model to also operate in undesired states to ensure that the controller detects the faults and acts accordingly (OBD diagnostics).

The aim of this paper is to introduce a range of faults in a multidomain/multi-physics engine model to demonstrate that it is capable of detecting and identifying these faults and of taking measures to limit their effect and/or to prevent further damage to the system.

The faults modelled are a leak in the air path of a turbocharged four-cylinder engine, a clogged injector in a naturally aspirated four-cylinder engine, a stretched timing chain in a naturally aspirated four-cylinder engine and a short-circuit in the TPS (Throttle Position Sensor) of a throttle body in a naturally aspirated fourcylinder engine.

The reason why we have chosen to focus on these faults in particular is because they allow to test components from all the domains involved in an engine model. Moreover, these are all faults commonly encountered in a real engine and ones that the ECU needs to be able to detect.

\section{Presentation of the engine models}

\subsection{Crank-angle resolved}

The engines used in this study come from the VeSyMA - Engines library developed by Claytex. They are all crank-angle resolved engine models with varying features and levels of detail depending on the area of interest.

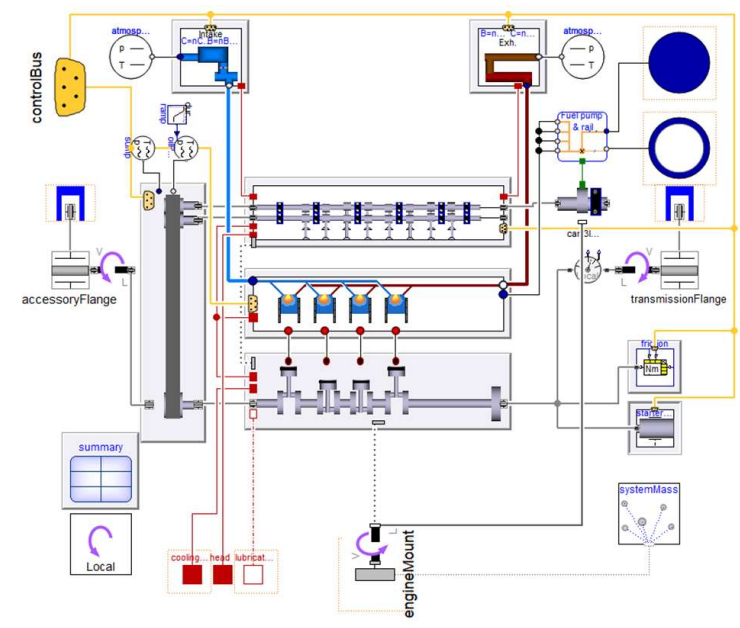

Figure 1. Crank-angle resolved engine model from VeSyMA - Engines.

The engine in Figure 1 is one of the most detailed versions available in the library [1] built referencing [2] and [3] mainly.

Some parameterisation adjustments have been made when necessary and simplifications when possible.

The mechanical systems can be reduced to 1dimensional models (air leak, clogged injector, faulty throttle). The stretched timing chain example needs 3dimensional mechanics since in the 1D one there is no mechanical link between the crankshaft and the cylinder head.

The surrogate model has been enabled (air leak, stretched timing belt, faulty throttle). The surrogate model is a subsystem that measures a set of physical quantities in cylinder 1 (intake air flow rate, cylinder pressure, etc.) and uses these values to drive ideal sources/actuators that are substituted to the physical 
fluid-based model in all the other engine cylinders. This helps to improve the model performance. The clogged injector example, however, needs all 3 cylinders to be enabled to allow for comparing their respective results and the variation in cylinder-to-cylinder performance.

\subsection{ECU (Engine Control Unit)}

The ECU is particularly important in this study since it is the subsystem that must be able to detect the faults and to react in relation to them.

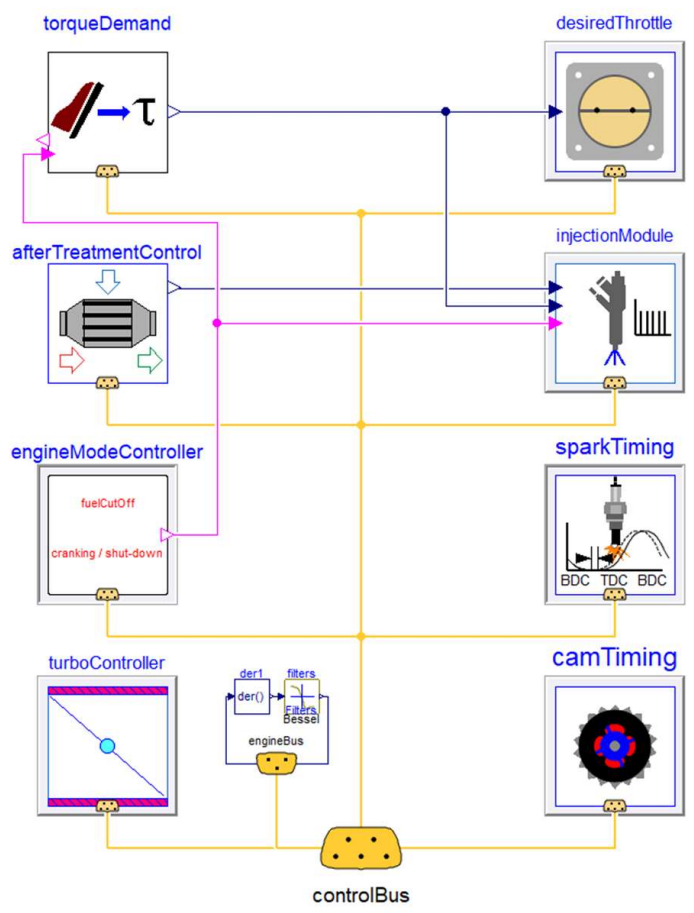

Figure 2. Engine Control Unit.Left-hand side modules from top to bottom: torque demand controller, aftertreatment devices controller, engine mode controller, turbocharger controller. Right-hand side modules, from top to bottom: throttle controller, injection controller, ignition controller, valvetrain controller.

Figure 2 shows the software version of an ECU. It is used to validate the engine model before using the real ECU and doing Hardware-in-the-Loop testing. It must rely entirely on information collected by the engine sensors to detect a fault, like a real ECU would do. It mostly uses PIDs and functions to control the engine actuators.

\section{The faults}

\subsection{Pneumatic - air leak}

A leak is introduced downstream of the intercooler and upstream of the throttle body.

It is modelled as an orifice connected to an infinite boundary at atmospheric pressure in parallel of the main air path.

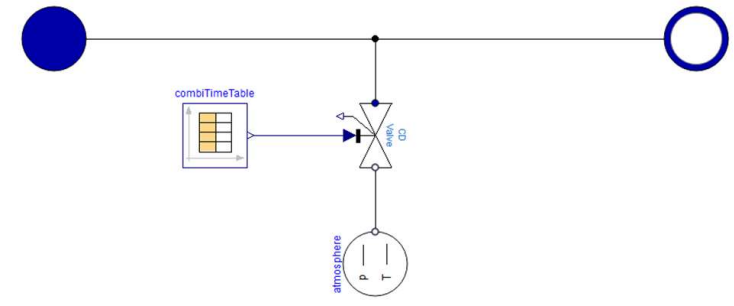

Figure 3. Air leak model with controllable orifice to ambient.

In Figure 3, port a (blue) is connected to the intercooler outlet and port $\bar{b}$ (white) is connected to the throttle inlet, in series. The valve discharge coefficient is entered in the time table, zero meaning no leak. This allows for the leakage to start at any moment. An external leak trigger can also be used.

\subsection{Hydraulic - clogged injector}

The injectors are modelled as ideal mass flow sources.

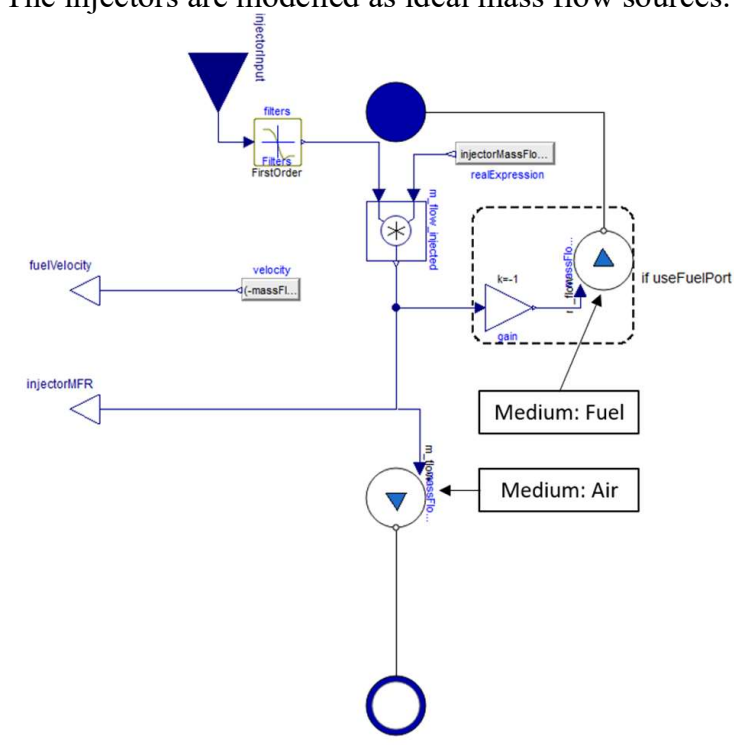

Figure 4. Injector model with optional fuel rail fluid port (filled blue round connector at top of diagram).

Two mass flow sources are used to separate the fuel path from the air path. The quantity of fuel to inject is calculated as the product of the nominal mass flow rate that the injector is capable of delivering by the injection pulse triggered by the ECU. This is the amount of fuel that the upper mass flow source in Figure 4 will remove (hence the negative gain) from the fuel line. The lower mass flow source will "inject" the same quantity into the combustion chamber.

The faulty injector is modelled in a similar way but the nominal mass flow the injector can deliver is determined by a time varying table. It is then possible to inject any fraction of what the injector should normally inject at any time. 


\subsection{Mechanical - stretched timing chain}

In this model the timing chain is essentially an ideal gear ratio (ratio $=0.5$, see Figure 5 ) located between the crankshaft and the camshaft flanges.

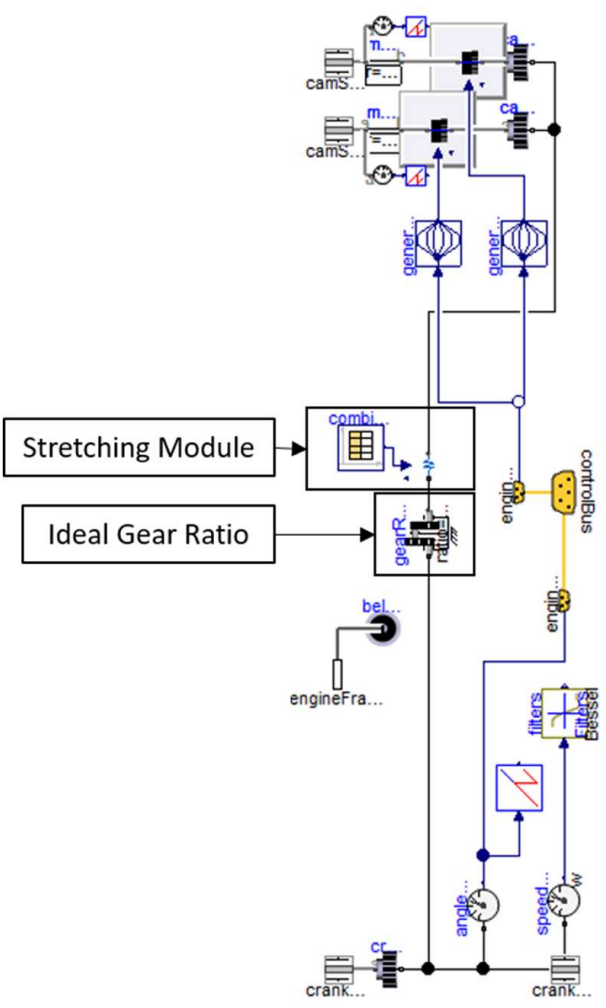

Figure 5. Timing chain module using ideal ratios with an angular offset to account for chain stretch.

The stretched timing chain module in Figure 5 is used to introduce a controlled degree of freedom between the crankshaft and the camshaft(s) angular.

In this case, we add an offset angle (between the crankshaft and the camshaft flanges) by means of a position actuator (see Figure 6). The chain is therefore still rigid but is stretched by a few millimetres.

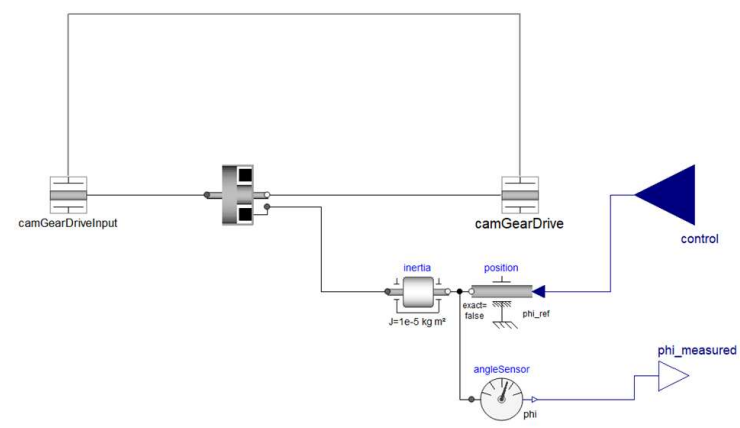

Figure 6. Compliance in the timing chain.

\subsection{Electrical - short-circuit in the throttle board/wrong sensor measurement}

The throttle body is a valve with varying discharge coefficient based on the plate opening angle (see 1 in Figure 7).

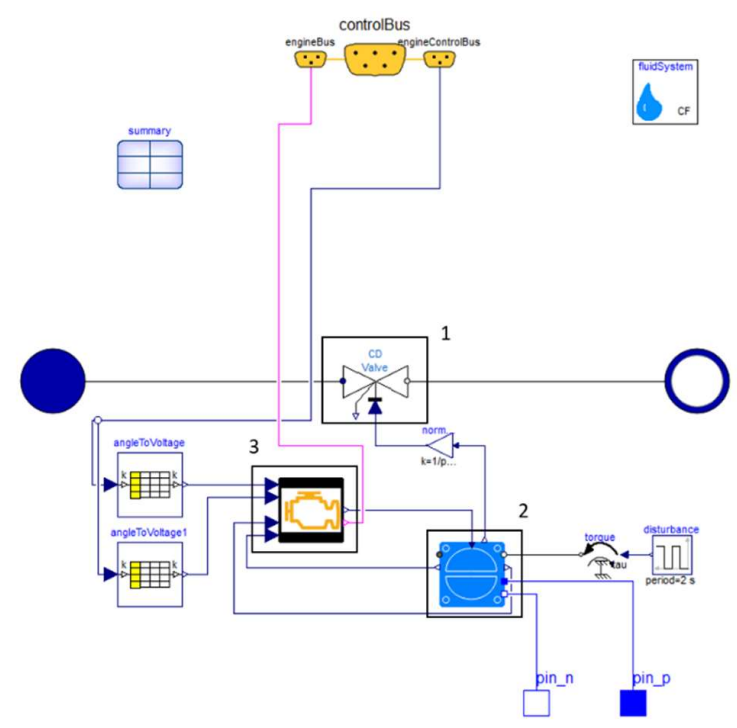

Figure 7. Throttle body with control board (1: pressure drop model, 2: mechanical system with potentiometerbased angle sensor, 3: electric board).

The throttle control board generates a current signal to drive the opening of the throttle plate (see section 4.4 for more info).

The throttle position sensor (Figure 8) uses a potentiometer to deliver a voltage as a function of the throttle opening angle.

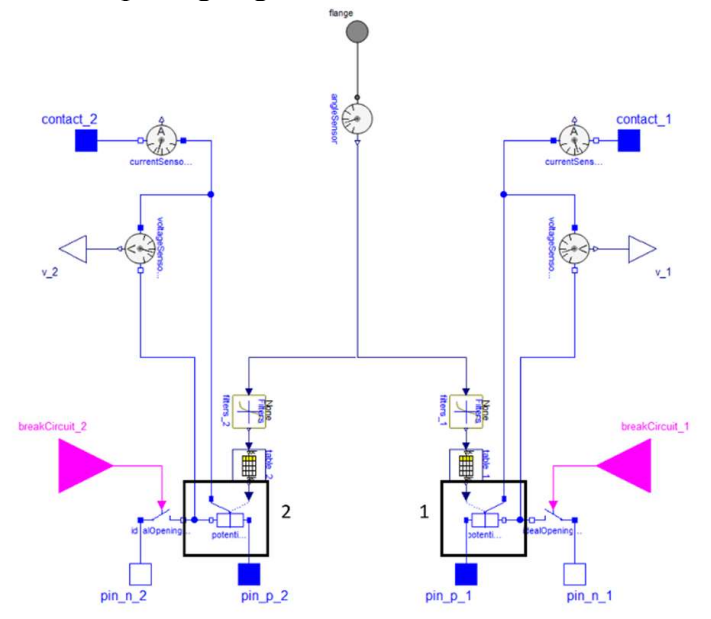

Figure 8. Throttle position sensor (1: potentiometer $1^{\text {st }}$ track, 2: potentiometer $2^{\text {nd }}$ track). 
A second potentiometer is used to provide a valid value in case the first one fails and delivers half the voltage of the other one. Comparing these two voltages is another way of detecting a failure. The short-circuit is induced in the TPS by means of a switch breaking the electrical circuit.

\section{Failure detection and simulation results}

\subsection{Air leak}

We run our engine coupled to a fixed inertia with wideopen throttle for $10 \mathrm{~s}$.

A leak corresponding to an orifice with a diameter of $0.04 \mathrm{~m}$ is introduced at $\mathrm{t}=2.5 \mathrm{~s}$. The leak diameter has been chosen rather large in order to see significant and short-term effects on the engine.

We compare the boost pressure from the plenum pressure sensor to the values mapped during normal operation. If they fall below a threshold, the leak is detected, and limp mode is activated which limits throttle opening to a small value.
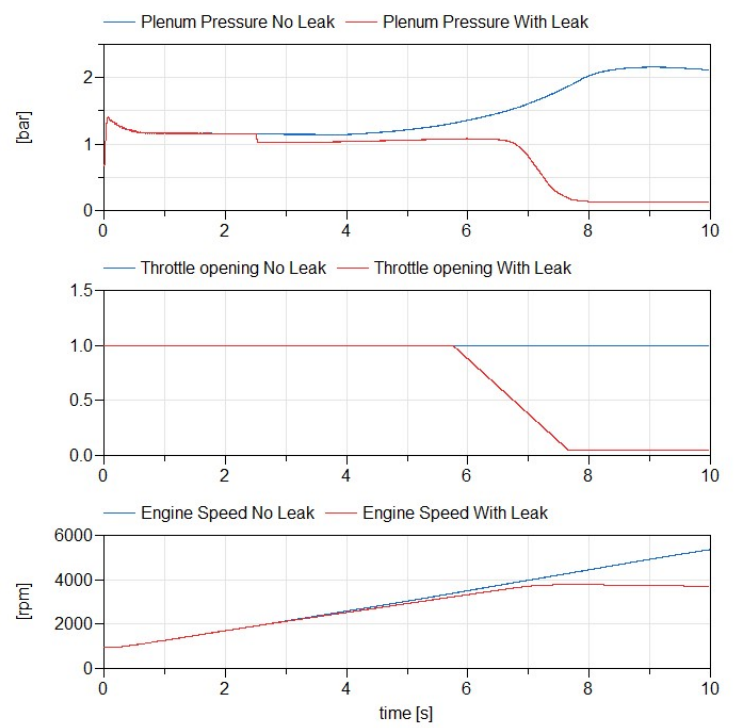

Figure 9. Plenum pressure, normalised throttle opening and engine speed under normal operation (blue) and with a leak (red).

We can see in Figure 9 that plenum pressure drops slightly when the leak starts. When it goes below the threshold at $\mathrm{t}=5.75 \mathrm{~s}$, limp mode gets activated and the maximum allowed normalised throttle opening is limited to 0.05 (i.e. $4.5 \mathrm{deg}$ ). Limiting throttle opening allows to keep engine torque below a certain threshold to prevent further damage. The value of this threshold depends on the engine manufacturer and type.

\subsection{Clogged injector}

The quantity of fuel injected by one of the injectors is limited for a few engine cycles to $25 \%$ of what the other injectors deliver.

The misfire detection monitor, which comprises of a set of logic blocks, compares the maximum crankshaft acceleration during every cycle to a value corresponding to normal operation based on an inferred torque for the set of given operating conditions. If a misfire is detected in the same cylinder during two consecutive cycles (can be changed to any number of consecutive cycles), it is considered a fault and the engine malfunction light goes on and limp mode gets activated. The detection monitor is not active during fast transients when the cylinder pressure varies a lot from one engine cycle to the next, like during aggressive tip-ins or tip-outs for example.
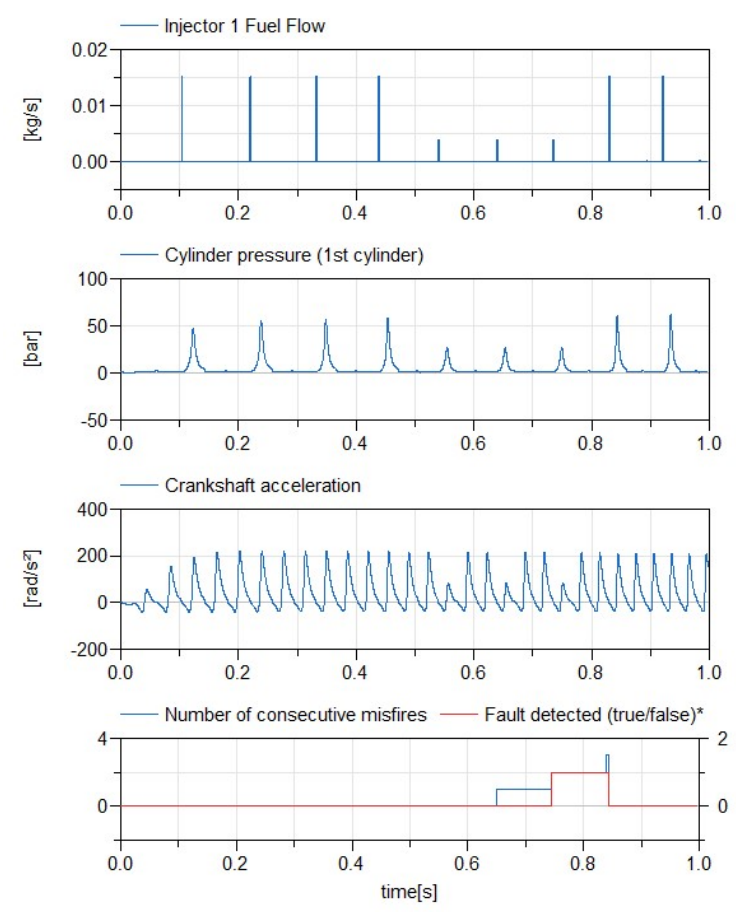

Figure 10: Injector fuel flow rate, cylinder pressure, crankshaft acceleration and number of consecutive misfires.

At about $5.5 \mathrm{~s}$, when the injector starts to inject less fuel than it should, the peak pressure in the cylinder decreases by more than $50 \%$. This has a direct effect on crankshaft acceleration (see subplot 3 in Figure 10 above). During the three consecutive cycles in which cylinder one misfires, we can observe irregularities on the crankshaft acceleration curve in figure 9 (at about $\mathrm{t}=5.5 \mathrm{~s}, \mathrm{t}=6.5 \mathrm{~s}$ and $\mathrm{t}=7.5 \mathrm{~s}$ ).

The acceleration of the crankshaft is computed from the crankshaft speed which is calculated from its angular position. The crankshaft angle is given by a crankshaft position sensor, this is then the physical quantity that will help the ECU to detect the abnormal behaviour. 


\subsection{Stretched timing chain}

The timing chain is allowed to stretch by a few millimetres causing a slight desynchronisation between the opening of the intake and exhaust valves and the pistons positions.

The ECU detects a mismatch between the angle given by the crankshaft and the camshaft position sensors.

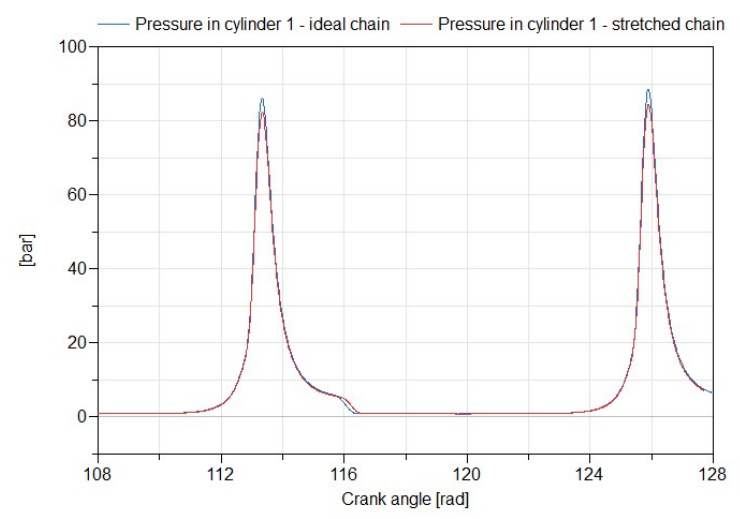

Figure 11: Pressure trace in cylinder 1 with an ideal chain (blue) and a stretched chain (red).

After a few cycles, the peak pressure reaches 88.4 bar when the chain is not stretched and the valve timing is ideal. It only reaches 84.4 bar $(4.5 \%$ drop) when the chain is stretched by 5 degrees. The pressure trace for cylinder 1 is plotted in Figure 11. Cylinder 1 has been chosen arbitrarily but the results would be the same in all the other cylinders. The reduction in cylinder pressure is due to a larger valve overlap that causes the combustion chamber to not fill in with air as much as it could. The fuel injection module in the ECU maintains the air-fuel-ratio to 14.67 which means that less fuel gets injected leading to a lower power output.

The reduction in cylinder pressure compared to the expected value for a given engine speed and throttle opening is a way for the ECU to detect the fault. Combined with a mismatch in readings from the crankshaft and camshaft position sensors, it allows to identify the cause of the problem.

\subsection{Faulty throttle body}

A short-circuit is introduced during the simulation.

When the driver presses the accelerator pedal, the pedal position sensor (which is similar to the throttle position sensor in Figure 8 ) transmits a normalised angle signal to the ECU and reads a voltage using a potentiometer. Depending on the engine operating conditions, the ECU will determine the correct throttle opening. To this throttle opening angle corresponds a voltage (volts) that the throttle position sensor will compare to the value the accelerator pedal position sensor measured.

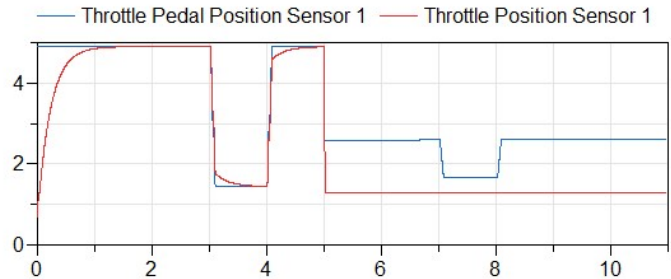

— Throttle Pedal Position Sensor 2 — Throttle Position Sensor 2

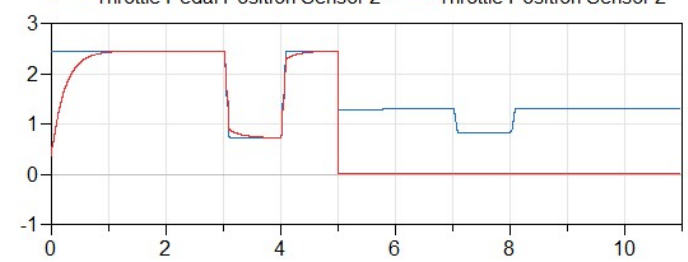

— Throttle opening

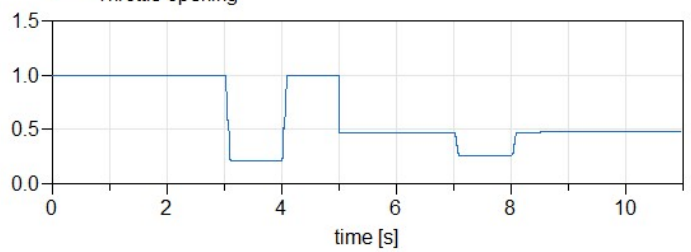

Figure 12: Comparison of the voltages from both channels of the throttle pedal position sensor and throttle position sensor. The bottom plot is throttle opening.

A short-circuit is introduced at time $=5 \mathrm{~s}$ in the second channel of the TPS which causes the voltage in the second channel to drop to 0 . At this time, the ECU goes in to safety mode and the electrical current to the throttle plate spindle magnet is cut to zero which effectively corresponds to a fast idle setting for the throttle plate.

\section{Conclusion and future work}

Faults have been introduced in a crank-angle resolved engine model. The results before and after the faults occur have been presented to highlight their impact on the engine behaviour.

It has been demonstrated that the soft ECU can detect them relying solely on the information coming from the sensors and that the engine model can therefore be used for controller calibration tasks.

The next steps are to export this engine model as an FMU to a real-time platform and to test it with a real ECU. To do so, we would have to make further simplifications to the model for it to achieve real-time performance.

\section{Bibliographic References}

[1] Dempsey M. Picarelli A. Investigating the MultiBody Dynamics of the Complete Powertrain System. Como, Italy: Proceedings 7 th Modelica Conference, 2009.

[2] Heywood, B. Internal Combustion Engine Fundamentals McGraw-Hill.

[3] Robert Bosch Gmbh Gasoline Engine Management Bentley Publishers 2006. 
